\title{
THE GENERALIZATION \\ OF THE DU BOIS-REYMOND LEMMA \\ FOR FUNCTIONS OF TWO VARIABLES \\ TO THE CASE OF PARTIAL DERIVATIVES OF ANY ORDER
}

\author{
DARIUSZ IDCZAK \\ Institute of Mathematics, Eódź University \\ Stefana Banacha 22, 90-238 Eódź, Poland
}

Abstract. In the paper, the generalization of the Du Bois-Reymond lemma for functions of two variables to the case of partial derivatives of any order is proved. Some application of this theorem to the coercive Dirichlet problem is given.

1. Introduction. To begin with, let us denote: $I=[0,1] \subset \mathbb{R}, P^{2}=[0,1] \times[0,1] \subset$ $\mathbb{R}^{2}$,

$$
\begin{aligned}
A C_{0}^{k, 2}\left(I, \mathbb{R}^{n}\right)= & \left\{h: I \rightarrow \mathbb{R}^{n} \mid h^{(i)} \text { is absolutely continuous on } I\right. \\
& \text { and } \left.h^{(i)}(0)=h^{(i)}(1)=0 \text { for } i=0, \ldots, k-1, h^{(k)} \in L^{2}\left(I, \mathbb{R}^{n}\right)\right\}
\end{aligned}
$$

for $k \geq 1$ and

$$
\begin{aligned}
A C_{0}^{(k, l), 2}\left(P^{2}, \mathbb{R}^{n}\right)=\{ & h: P^{2} \rightarrow \mathbb{R}^{n} \mid \frac{\partial^{i+j} h}{\partial x^{i} \partial y^{j}} \text { is absolutely continuous on } P^{2} \\
& \text { and }\left|\frac{\partial^{i+j} h}{\partial x^{i} \partial y^{j}}\right|_{\partial P^{2}} \equiv 0 \text { for } i=0, \ldots, k-1, j=0, \ldots, l-1, \\
& \left.\frac{\partial^{k+l} h}{\partial x^{k} \partial y^{l}} \in L^{2}\left(P^{2}, \mathbb{R}^{n}\right)\right\}
\end{aligned}
$$

for $k \geq 1, l \geq 1$.

The following theorem plays a very important role in the classical variational calculus and in the theory of ordinary differential equations.

1991 Mathematics Subject Classification: Primary 49E10; Secondary $26 B 30$.

The paper is in final form and no version of it will be published elsewhere. 
THEOREM 1.1. If $v \in L^{2}\left(I, \mathbb{R}^{n}\right)$ and

$$
\int_{I} v(t) h^{\prime}(t) d t=0
$$

for any $h \in A C_{0}^{1,2}\left(I, \mathbb{R}^{n}\right)$, then there exists a constant $c_{0} \in \mathbb{R}^{n}$ such that

$$
v(t)=c_{0}
$$

for $t \in I$ a.e.

In paper [4], the following generalization of the above theorem to the case of derivatives of order $k \geq 2$ is given:

THEOREM 1.2. If $v \in L^{2}\left(I, \mathbb{R}^{n}\right)$ and

$$
\int_{I} v(t) h^{(k)}(t) d t=0
$$

for any $h \in A C_{0}^{k, 2}\left(I, \mathbb{R}^{n}\right)$, then there exists constants $c_{0}, \ldots, c_{k-1} \in \mathbb{R}^{n}$ such that

$$
v(t)=c_{k-1} t^{k-1}+\cdots+c_{1} t+c_{0}
$$

for $t \in I$ a.e.

In paper [5], the following generalization of theorem 1.1 to the case of functions of two variables is proved:

THEOREM 1.3. If $v \in L^{2}\left(P^{2}, \mathbb{R}^{n}\right)$ and

$$
\iint_{P^{2}} v(s, t) \frac{\partial^{2} h}{\partial x \partial y}(s, t) d s d t=0
$$

for any $h \in A C_{0}^{(1,1), 2}\left(P^{2}, \mathbb{R}^{n}\right)$, then there exists functions $b_{0}(\cdot), c_{0}(\cdot) \in L^{2}\left(I, \mathbb{R}^{n}\right)$ and a constant $a_{00} \in \mathbb{R}^{n}$, such that

$$
v(s, t)=b_{0}(t)+c_{0}(s)+a_{00}
$$

for $(s, t) \in P^{2}$ a.e.

In our paper we shall prove a generalization of theorem 1.3 to the case of partial derivatives of higher orders.

2. Main result. The main result of the paper is

THEOREM 2.1. If $v \in L^{2}\left(P^{2}, \mathbb{R}^{n}\right)$ and

$$
\iint_{P^{2}} v(s, t) \frac{\partial^{k+l} h}{\partial x^{k} \partial y^{l}}(s, t) d s d t=0
$$

for any $h \in A_{0}^{(k, l), 2}\left(P^{2}, \mathbb{R}^{n}\right)$, then there exist functions $b_{i}(\cdot) \in L^{2}\left(I, \mathbb{R}^{n}\right), i=0, \ldots, k-1$, $c_{j}(\cdot) \in L^{2}\left(I, \mathbb{R}^{n}\right), j=0, \ldots, l-1$ and constants $a_{i j} \in \mathbb{R}^{n}, i=0, \ldots, k-1, j=0, \ldots, l-1$, such that

$$
v(s, t)=\sum_{i=0}^{k-1} \sum_{j=0}^{l-1} a_{i j} s^{i} t^{j}+\sum_{i=0}^{k-1} b_{i}(t) s^{i}+\sum_{j=0}^{l-1} c_{j}(s) t^{j}
$$

for $(s, t) \in P^{2}$ a.e. 
Proof. Let us observe that

$$
\begin{aligned}
& A C_{0}^{(k, l), 2}\left(P, \mathbb{R}^{n}\right)=\left\{h: P^{2} \rightarrow \mathbb{R}^{n} \mid \text { there exists a function } l \in L^{2}\left(P^{2}, \mathbb{R}^{n}\right)\right. \text { such that } \\
& h\left(x_{1}, y_{1}\right)= \int_{0}^{x_{1}} \int_{0}^{x_{2}} \ldots \int_{0}^{x_{k}} \int_{0}^{y_{1}} \int_{0}^{y_{2}} \ldots \int_{0}^{y_{l}} l(s, t) d t d y_{l-1} \ldots d y_{2} d s d x_{k-1} \ldots d x_{2}, \quad \text { for }\left(x_{1}, y_{1}\right) \in P^{2}, \\
& \int_{0}^{1} \int_{0}^{x_{2}} \ldots \int_{0}^{x_{k-i}} \int_{0}^{y_{1}} \ldots \int_{0}^{y_{l-j}} l=0 \text { for } y_{1} \in I, \\
& \int_{0}^{x_{1}} \ldots \int_{0}^{x_{k-i}} \int_{0}^{1} \int_{0}^{y_{2}} \ldots \int_{0}^{y_{l-j}} l=0 \text { for } x_{1} \in I, \\
&i=0, \ldots, k-1, j=0, \ldots, l-1\} .
\end{aligned}
$$

It is easy to see that

$$
\int_{0}^{1} \int_{0}^{x_{2}} \cdots \int_{0}^{x_{k-i}} \int_{0}^{y_{1}} \cdots \int_{0}^{y_{l-j}} l=0
$$

for any $y_{1} \in I$ if and only if

$$
\int_{0}^{1} \int_{0}^{x_{2}} \ldots \int_{0}^{x_{k-i}} l(s, t) d s d x_{k-i} \ldots d x_{2}=0
$$

for $t \in I$ a.e. Analogously,

$$
\int_{0}^{x_{1}} \cdots \int_{0}^{x_{k-i}} \int_{0}^{1} \int_{0}^{y_{2}} \cdots \int_{0}^{y_{l-j}} l=0
$$

for any $x_{1} \in I$ if and only if

$$
\int_{0}^{1} \int_{0}^{y_{2}} \cdots \int_{0}^{y_{l-j}} l(s, t) d t d y_{l-j} \ldots d y_{2}=0
$$

for $s \in I$ a.e. So, we may write

$$
\begin{aligned}
& A C_{0}^{(k, l), 2}\left(P^{2}, \mathbb{R}^{n}\right)=\left\{h: P^{2} \rightarrow \mathbb{R}^{n} \mid \text { there exists a function } l \in L^{2}\left(P^{2}, \mathbb{R}^{n}\right)\right. \text { such that } \\
& h\left(x_{1}, y_{1}\right)=\int_{0}^{x_{1}} \ldots \int_{0}^{x_{k}} \int_{0}^{y_{1}} \ldots \int_{0}^{y_{l}} l \text { for }\left(x_{1}, y_{1}\right) \in P^{2}, \\
& \int_{0}^{1} \int_{0}^{x_{2}} \cdots \int_{0}^{x_{k-i}} l(s, t) d s \ldots d x_{2}=0 \text { for } t \in I \text { a.e., } \\
& \int_{0}^{1} \int_{0}^{y_{2}} \ldots \int_{0}^{y_{l-j}} l(s, t) d t \ldots d y_{2}=0 \text { for } s \in I \text { a.e. }, \\
&i=0, \ldots, k-1, j=0, \ldots, l-1\} .
\end{aligned}
$$

Now, we shall show that, for any functions $b_{i}(\cdot) \in L^{2}\left(P^{2}, \mathbb{R}^{n}\right), i=0, \ldots, k-1, c_{j}(\cdot) \in$ $L^{2}\left(P^{2}, \mathbb{R}^{n}\right), j=0, \ldots, l-1$, and constants $a_{i j} \in \mathbb{R}^{n}, i=0, \ldots, k-1, j=0, \ldots, l-1$, we have

$$
\iint_{P^{2}}\left(\sum_{i=0}^{k-1} \sum_{j=0}^{l-1} a_{i j} s^{i} t^{j}\right) \frac{\partial^{k+l} h}{\partial x^{k} \partial y^{l}}(s, t) d s d t=0
$$




$$
\begin{aligned}
& \iint_{P^{2}}\left(\sum_{i=0}^{k-1} b_{i}(t) s^{i}\right) \frac{\partial^{k+l} h}{\partial x^{k} \partial y^{l}}(s, t) d s d t=0, \\
& \iint_{P^{2}}\left(\sum_{j=0}^{l-1} c_{j}(s) t^{j}\right) \frac{\partial^{k+l} h}{\partial x^{k} \partial y^{l}}(s, t) d s d t=0,
\end{aligned}
$$

for any $h \in A C_{0}^{(k, l), 2}\left(P^{2}, \mathbb{R}^{n}\right)$. Of course, it is sufficient to prove that, for any $i=0, \ldots$, $k-1, b_{i}(\cdot) \in L^{2}\left(I, \mathbb{R}^{n}\right)$,

$$
\int_{0}^{1} \int_{0}^{1} b_{i}(t) s^{i} \frac{\partial^{k+l} h}{\partial x^{k} \partial y^{l}}(s, t) d s d t=0,
$$

and, for any $j=0, \ldots, l-1, c_{j}(\cdot) \in L^{2}\left(I, \mathbb{R}^{n}\right)$,

$$
\int_{0}^{1} \int_{0}^{1} c_{j}(s) t^{j} \frac{\partial^{k+l} h}{\partial x^{k} \partial y^{l}}(s, t) d s d t=0 .
$$

Indeed, we have

$$
\begin{aligned}
\int_{0}^{1} \int_{0}^{1} b_{i}(t) s^{i} \frac{\partial^{k+l} h}{\partial x^{k} \partial y^{l}}(s, t) d s d t \\
=\int_{0}^{1} b_{i}(t) \int_{0}^{1} s^{i} \frac{\partial}{\partial x}\left(\frac{\partial^{k-1+l} h}{\partial x^{k-1} \partial y^{l}}\right)(s, t) d s d t \\
=\int_{0}^{1} b_{i}(t)\left(\left.s^{i} \frac{\partial^{k-1+l} h}{\partial x^{k-1} \partial y^{l}}(s, t)\right|_{s=0} ^{s=1}-\int_{0}^{1} i s^{i-1} \frac{\partial^{k-1+l} h}{\partial x^{k-1} \partial y^{l}}(s, t) d s\right) d t \\
=\int_{0}^{1} b_{i}(t)\left(\frac{\partial}{\partial y}\left(\frac{\partial^{k-1+l-1} h}{\partial x^{k-1} \partial y^{l-1}}\right)(1, t)-i \int_{0}^{1} s^{i-1} \frac{\partial^{k-1+l} h}{\partial x^{k-1} \partial y^{l}}(s, t) d s\right) d t \\
=-i \int_{0}^{1} b_{i}(t) \int_{0}^{1} s^{i-1} \frac{\partial^{k-1+l} h}{\partial x^{k-1} \partial y^{l}}(s, t) d s d t=\ldots \\
=(-1)^{i} i(i-1) \cdot \ldots \cdot 1 \int_{0}^{1} b_{i}(t) \int_{0}^{1} \frac{\partial^{k-i+l} h}{\partial x^{k-i} \partial y^{l}}(s, t) d s d t \\
=(-1)^{i} i ! \int_{0}^{1} b_{i}(t)\left(\frac{\partial^{k-i-1+l} h}{\partial x^{k-i-1} \partial y^{l}}(1, t)-\frac{\partial^{k-i-1+l} h}{\partial x^{k-i-1} \partial y^{l}}(0, t)\right) d t \\
=(-1)^{i} i ! \int_{0}^{1} b_{i}(t)\left(\frac{\partial}{\partial y}\left(\frac{\partial^{k-i-1+l-1} h}{\partial x^{k-i-1} \partial y^{l-1}}\right)(1, t)-\frac{\partial}{\partial y}\left(\frac{\partial^{k-i-1+l-1} h}{\partial x^{k-i-1} \partial y^{l-1}}\right)(0, t)\right) d t \\
=(-1)^{i} i ! \int_{0}^{1} b_{i}(t)(0-0) d t=0 .
\end{aligned}
$$

In a analogous way one can obtain (6).

So, from (1)-(4) it follows that, for any functions $b_{i}(\cdot) \in L^{2}\left(I, \mathbb{R}^{n}\right), i=0, \ldots, k-1$, $c_{j}(\cdot) \in L^{2}\left(I, \mathbb{R}^{n}\right), j=0, \ldots, l-1$, and constants $a_{i j} \in \mathbb{R}^{n}, i=0, \ldots, k-1, j=0, \ldots, l-1$, the function

$$
\widetilde{v}(s, t)=v(s, t)-\sum_{i=0}^{k-1} \sum_{j=0}^{l-1} a_{i j} s^{i} t^{j}-\sum_{i=0}^{k-1} b_{i}(t) s^{i}-\sum_{j=0}^{l-1} c_{j}(s) t^{j}
$$


integrable on $P^{2}$ with power 2 satisfies the condition of type (1), i.e.

$$
\iint_{P^{2}} \widetilde{v}(s, t) \frac{\partial^{k+l} h}{\partial x^{k} \partial y^{l}}(s, t) d s d t=0
$$

for any $h \in A C_{0}^{(k, l), 2}\left(P^{2}, \mathbb{R}^{n}\right)$.

Consequently, to end the proof, it is sufficient to show that there exist functions $b_{i}(\cdot) \in L^{2}\left(I, \mathbb{R}^{n}\right), i=0, \ldots, k-1, c_{j}(\cdot) \in L^{2}\left(I, \mathbb{R}^{n}\right), j=0, \ldots, l-1$, and constants $a_{i j} \in \mathbb{R}^{n}, i=0, \ldots, k-1, j=0, \ldots, l-1$, such that the function given by the formula

$$
h_{0}\left(x_{1}, y_{1}\right)=\int_{0}^{x_{1}} \cdots \int_{0}^{x_{k}} \int_{0}^{y_{1}} \cdots \int_{0}^{y_{l}} \widetilde{v}
$$

for $\left(x_{1}, y_{1}\right) \in P^{2}$, where $\widetilde{v}$ is a function of form (7), is an element of $A C_{0}^{(k, l), 2}\left(P^{2}, \mathbb{R}^{n}\right)$.

The form of the function $h_{0}$ and the integrability of $\widetilde{v}$ imply that it suffices to show the existence of functions $b_{i}(\cdot) \in L^{2}\left(I, \mathbb{R}^{n}\right), i=0, \ldots, k-1, c_{j}(\cdot) \in L^{2}\left(I, \mathbb{R}^{n}\right), j=$ $0, \ldots, l-1$, and constants $a_{i j} \in \mathbb{R}^{n}, i=0, \ldots, k-1, j=0, \ldots, l-1$, such that

$$
\int_{0}^{1} \int_{0}^{x_{2}} \ldots \int_{0}^{x_{k-i}} \widetilde{v}(s, t) d s d x_{k-i} \ldots d x_{2}=0
$$

for $t \in I$ a.e., $i=0, \ldots, k-1$, and

$$
\int_{0}^{1} \int_{0}^{y_{2}} \ldots \int_{0}^{y_{l-j}} d t d y_{l-j} \ldots d y_{2}=0
$$

for $s \in I$ a.e., $j=0, \ldots, l-1$.

System (8)-(9) may be written down in the form

$$
\begin{aligned}
\int_{0}^{1} \int_{0}^{x_{2}} \ldots \int_{0}^{x_{k-\tilde{i}}} v & -\sum_{i=0}^{k-1} \sum_{j=0}^{l-1} a_{i j} t^{j} \int_{0}^{1} \int_{0}^{x_{2}} \ldots \int_{0}^{x_{k-\tilde{i}}} s^{i} \\
& -\sum_{i=0}^{k-1} b_{i}(t) \int_{0}^{1} \int_{0}^{x_{2}} \ldots \int_{0}^{x_{k-\tilde{i}}} s^{i}-\sum_{j=0}^{l-1} t^{j} \int_{0}^{1} \int_{0}^{x_{2}} \ldots \int_{0}^{x_{k-\tilde{i}}} c_{j}(s)=0
\end{aligned}
$$

for $t \in I$ a.e., $\tilde{i}=0, \ldots, k-1$, and

$$
\begin{aligned}
\int_{0}^{1} \int_{0}^{y_{2}} \ldots \int_{0}^{y_{l-\tilde{j}}} v & -\sum_{i=0}^{k-1} \sum_{j=0}^{l-1} a_{i j} s^{i} \int_{0}^{1} \int_{0}^{y_{2}} \ldots \int_{0}^{y_{l-\tilde{j}}} t^{j} \\
& -\sum_{i=0}^{k-1} s^{i} \int_{0}^{1} \int_{0}^{y_{2}} \cdots \int_{0}^{y_{l-\tilde{j}}} b_{i}(t)-\sum_{j=0}^{l-1} c_{j}(s) \int_{0}^{1} \int_{0}^{y_{2}} \cdots \int_{0}^{y_{l-\tilde{j}}} t^{j}=0
\end{aligned}
$$

for $s \in I$ a.e., $\tilde{j}=0, \ldots, l-1$.

It is easy to see that it is enough to find functions $b_{i}(\cdot)=\left(b_{i}^{1}(\cdot), \ldots, b_{i}^{n}(\cdot)\right) \in$ $L^{2}\left(I, \mathbb{R}^{n}\right), i=0, \ldots, k-1, c_{j}(\cdot)=\left(c_{j}^{1}(\cdot), \ldots, c_{j}^{n}(\cdot)\right) \in L^{2}\left(I, \mathbb{R}^{n}\right), j=0, \ldots, l-1$, and constants $a_{i j}=\left(a_{i j}^{1}, \ldots, a_{i j}^{n}\right) \in \mathbb{R}^{n}, i=0, \ldots, k-1, j=0, \ldots, l-1$, that satisfy the following systems:

$$
S_{k, s} \circ\left[\begin{array}{c}
b_{0}(t) \\
\vdots \\
b_{k-1}(t)
\end{array}\right]=\left[\begin{array}{c}
\int_{0}^{1} \int_{0}^{x_{2}} \cdots \int_{0}^{x_{k}} v(s, t) \\
\vdots \\
\int_{0}^{1} v(s, t)
\end{array}\right] \quad \text { for } t \in I \text { a.e. }
$$




$$
S_{k, s} \circ\left[\begin{array}{c}
a_{0 j} \\
\vdots \\
a_{k-1 j}
\end{array}\right]=\left[\begin{array}{c}
-\int_{0}^{1} \int_{0}^{x_{2}} \ldots \int_{0}^{x_{k}} c_{j}(s) \\
\vdots \\
-\int_{0}^{1} c_{j}(s)
\end{array}\right] \quad \text { for } j=0, \ldots, l-1,
$$

$$
\begin{aligned}
& S_{l, t} \circ\left[\begin{array}{c}
c_{0}(s) \\
\vdots \\
c_{l-1}(s)
\end{array}\right]=\left[\begin{array}{c}
\int_{0}^{1} \int_{0}^{y_{2}} \cdots \int_{0}^{y_{l}} v(s, t) \\
\vdots \\
\int_{0}^{1} v(s, t)
\end{array}\right] \quad \text { for } s \in I \text { a.e., } \\
& S_{l, t} \circ\left[\begin{array}{c}
a_{i 0} \\
\vdots \\
a_{i l-1}
\end{array}\right]=\left[\begin{array}{c}
-\int_{0}^{1} \int_{0}^{y_{2}} \ldots \int_{0}^{y_{l}} b_{i}(t) \\
\vdots \\
-\int_{0}^{1} b_{i}(t)
\end{array}\right] \quad \text { for } i=0, \ldots, k-1 \text {, }
\end{aligned}
$$

where

$$
S_{k, s}=\left[\begin{array}{ccc}
\int_{0}^{1} \int_{0}^{x_{2}} \ldots \int_{0}^{x_{k}} s^{0} & \ldots & \int_{0}^{1} \int_{0}^{x_{2}} \ldots \int_{0}^{x_{k}} s^{k-1} \\
\vdots & & \vdots \\
\int_{0}^{1} s^{0} & \ldots & \int_{0}^{1} s^{k-1}
\end{array}\right]
$$

and, analogously,

$$
S_{l, t}=\left[\begin{array}{ccc}
\int_{0}^{1} \int_{0}^{y_{2}} \ldots \int_{0}^{y_{l}} t^{0} & \ldots & \int_{0}^{1} \int_{0}^{y_{2}} \ldots \int_{0}^{y_{l}} t^{l-1} \\
\vdots & & \vdots \\
\int_{0}^{1} t^{0} & \ldots & \int_{0}^{1} t^{l-1}
\end{array}\right] .
$$

In an elementary way one can show that $\operatorname{det} S_{k, s} \neq 0$. So, for any $t \in I$ such that the function $v(t, \cdot)$ is integrable on $I$, there exists a unique solution $\left(b_{0}(t), \ldots, b_{k-1}(t)\right)$ of system (10). From the Cramer formulae it follows that $b_{i}(\cdot) \in L^{2}\left(I, \mathbb{R}^{n}\right), i=0, \ldots, k-1$.

In an analogous way we obtain functions $\left(c_{0}(t), \ldots, c_{l-1}(t)\right)$ that are integrable on $I$ with power 2 and satisfy system (12) for any $s \in I$, such that the function $v(\cdot, s)$ is integrable on $I$.

So, to end the proof we must demonstrate that the solutions of systems $\left(11_{0}\right)-\left(11_{l-1}\right)$ and $\left(13_{0}\right)-\left(13_{k-1}\right)$ are identical.

Let us introduce some notations.

$S_{k, s}^{i}\left(\begin{array}{c}d_{k} \\ \vdots \\ d_{1}\end{array}\right)$ - the matrix $S_{k, s}$ with the column $i$ replaced by the vector $\left(\begin{array}{c}d_{k} \\ \vdots \\ d_{1}\end{array}\right)$,

$S_{l, t}^{j}\left(\begin{array}{c}e_{l} \\ \vdots \\ e_{1}\end{array}\right)$ - the matrix $S_{l, t}$ with the column $j$ replaced by the vector $\left(\begin{array}{c}e_{l} \\ \vdots \\ e_{1}\end{array}\right)$,

$\left(S_{k, s}\right)_{w, i}$ - the minor $(w, i)$ of $S_{k, s}$,

$\left(S_{l, t}\right)_{z, j}$ - the minor $(z, j)$ of $S_{l, t}$,

$\left(\bar{a}_{i j}, i=0, \ldots, k-1, j=0, \ldots, l-1\right)$ - the solution of system $\left(11_{0}\right)-\left(11_{l-1}\right)$,

$\left(\overline{\bar{a}}_{i j}, i=0, \ldots, k-1, j=0, \ldots, l-1\right)$ - the solution of system $\left(13_{0}\right)-\left(13_{k-1}\right)$. 
Let us fix numbers $i \in\{0, \ldots, k-1\}, j \in\{0, \ldots, l-1\}, \alpha \in\{1, \ldots, n\}$. We have

$$
\begin{aligned}
\bar{a}_{i j}^{\alpha}= & \left|S_{k, s}^{i}\left(\begin{array}{c}
-\int_{0}^{1} \int_{0}^{x_{2}} \ldots \int_{0}^{x_{k}} c_{j}^{\alpha}(s) \\
\vdots \\
-\int_{0}^{1} c_{j}^{\alpha}(s)
\end{array}\right)\right| /\left|S_{k, s}\right| \\
= & \left((-1) \sum_{w=0}^{k-1}(-1)^{w+i}\left|\left(S_{k, s}\right)_{w, i}\right| \int_{0}^{1} \int_{0}^{x_{2}} \cdots \int_{0}^{x_{k-w}} c_{j}^{\alpha}(s)\right) /\left|S_{k, s}\right| \\
= & (-1) \sum_{w=0}^{k-1}(-1)^{w+i}\left|\left(S_{k, s}\right)_{w, i}\right| \\
& \left.\cdot\left(\left|S_{l, t}^{j}\left(\begin{array}{c}
\int_{0}^{1} \ldots \int_{0}^{x_{k-w}} \int_{0}^{1} \ldots \int_{0}^{y_{l}} v^{\alpha}(s, t) \\
\vdots \\
\int_{0}^{1} \ldots \int_{0}^{x_{k-w}} \int_{0}^{1} v^{\alpha}(s, t)
\end{array}\right)\right| /\left|S_{l, t}\right|\right)\right) /\left|S_{k, s}\right| \\
= & \left((-1) /\left(\left|S_{k, s}\right|\left|S_{l, t}\right|\right)\right) \sum_{w=0}^{k-1}(-1)^{w+i}\left|\left(S_{k, s}\right)_{w, i}\right| \\
& \cdot \sum_{z=0}^{l-1}(-1)^{z+j}\left|\left(S_{l, t}\right)_{z, j}\right| \int_{0}^{1} \ldots \int_{0}^{x_{k-w}} \int_{0}^{1} \ldots \int_{0}^{y_{l-z}} v^{\alpha}(s, t) \\
= & \left((-1) /\left(\left|S_{k, s}\right|\left|S_{l, t}\right|\right)\right) \sum_{w=0}^{k-1} \sum_{z=0}^{l-1}(-1)^{w+i+z+j}\left|\left(S_{k, s}\right)_{w, i}\right|\left|\left(S_{l, t}\right)_{z, j}\right| \\
& \cdot \int_{0}^{1} \ldots \int_{0}^{x_{k-w}} \int_{0}^{1} \ldots \int_{0}^{y_{l-z}} v^{\alpha}(s, t) .
\end{aligned}
$$

In an analogous way we obtain

$$
\begin{aligned}
\overline{\bar{a}}_{i j}^{\alpha}= & \left|S_{l, t}^{j}\left(\begin{array}{c}
-\int_{0}^{1} \int_{0}^{y_{2}} \cdots \int_{0}^{y_{l}} b_{i}^{\alpha}(t) \\
\vdots \\
-\int_{0}^{1} b_{i}^{\alpha}(t)
\end{array}\right)\right| /\left|S_{l, t}\right| \\
= & \left((-1) \sum_{z=0}^{l-1}(-1)^{z+j}\left|\left(S_{l, t}\right)_{z, j}\right| \int_{0}^{1} \int_{0}^{y_{2}} \cdots \int_{0}^{y_{l-z}} b_{i}^{\alpha}(t)\right) /\left|S_{l, t}\right| \\
= & \left((-1) \sum_{z=0}^{l-1}(-1)^{z+j}\left|\left(S_{l, t}\right)_{z, j}\right|\right. \\
& \left.\cdot\left(\left|S_{k, s}^{i}\left(\begin{array}{c}
\int_{0}^{1} \ldots \int_{0}^{y_{l-z}} \int_{0}^{1} \ldots \int_{0}^{x_{k}} v^{\alpha}(s, t) \\
\vdots \\
\int_{0}^{1} \ldots \int_{0}^{y_{l-z}} \int_{0}^{1} v^{\alpha}(s, t)
\end{array}\right)\right| /\left|S_{k, s}\right|\right)\right) /\left|S_{l, t}\right| \\
= & \left((-1) /\left(\left|S_{l, t}\right|\left|S_{k, s}\right|\right)\right) \sum_{z=0}^{l-1}(-1)^{z+j}\left|\left(S_{l, t}\right)_{z, j}\right| \sum_{w=0}^{k-1}(-1)^{w+i}\left|\left(S_{k, s}\right)_{w, i}\right| \\
& \cdot \int_{0}^{1} \ldots \int_{0}^{y_{l-z}} \int_{0}^{1} \ldots \int_{0}^{x_{k-w}} v^{\alpha}(s, t)
\end{aligned}
$$


So, $\bar{a}_{i j}^{\alpha}=\overline{\bar{a}}_{i j}^{\alpha}$ and the proof is completed.

$$
\begin{aligned}
= & \left((-1) /\left(\left|S_{l, t}\right|\left|S_{k, s}\right|\right)\right) \sum_{z=0}^{l-1} \sum_{w=0}^{k-1}(-1)^{z+j+w+i}\left|\left(S_{l, t}\right)_{z, j}\right|\left|\left(S_{k, s}\right)_{w, i}\right| \\
& \cdot \int_{0}^{1} \ldots \int_{0}^{y_{l-z}} \int_{0}^{1} \ldots \int_{0}^{x_{k-w}} v^{\alpha}(s, t) .
\end{aligned}
$$

Remark. From the above proof it follows that

$$
\begin{gathered}
a_{i j}^{\alpha}=\left((-1) /\left(\left|S_{k, s}\right|\left|S_{l, t}\right|\right)\right) \\
\cdot \sum_{w=0}^{k-1} \sum_{z=0}^{l-1}(-1)^{w+i+z+j}\left|\left(S_{k, s}\right)_{w, i}\right|\left|\left(S_{l, t}\right)_{z, j}\right| \int_{0}^{1} \ldots \int_{0}^{x_{k-w}} \int_{0}^{1} \ldots \int_{0}^{y_{l-z}} v^{\alpha}(s, t)
\end{gathered}
$$

for $i=0, \ldots, k-1, j=0, \ldots, l-1, \alpha=1, \ldots, n$,

$$
b_{i}^{\alpha}(t)=\left(1 /\left|S_{k, s}\right|\right)\left|S_{k, s}^{i}\left(\begin{array}{c}
\int_{0}^{1} \int_{0}^{x_{2}} \cdots \int_{0}^{x_{k}} v^{\alpha}(s, t) d s \\
\vdots \\
\int_{0}^{1} v^{\alpha}(s, t) d s
\end{array}\right)\right|
$$

for $t \in[0,1]$ a.e., $i=0, \ldots, k-1, \alpha=1, \ldots, n$, and

$$
c_{j}^{\alpha}(s)=\left(1 /\left|S_{l, t}\right|\right)\left|S_{l, t}^{j}\left(\begin{array}{c}
\int_{0}^{1} \int_{0}^{y_{2}} \cdots \int_{0}^{y_{l}} v^{\alpha}(s, t) d s \\
\vdots \\
\int_{0}^{1} v^{\alpha}(s, t) d s
\end{array}\right)\right|
$$

for $s \in[0,1]$ a.e., $j=0, \ldots, l-1, \alpha=1, \ldots, n$.

3. Further generalizations. In monograph [3], the following generalization of Theorem 1.1 is proved:

TheOREM 3.1. If $v \in L^{2}\left(I, \mathbb{R}^{n}\right)$ and $w \in L^{1}\left(I, \mathbb{R}^{n}\right)$ are such that

$$
\int_{I} v(t) h^{\prime}(t) d t=-\int_{I} w(t) h(t) d t
$$

for any $h \in A C_{0}^{1,2}\left(I, \mathbb{R}^{n}\right)$, then there exists a constant $c_{0} \in \mathbb{R}^{n}$ such that

$$
v(t)=\int_{0}^{t} w(s) d s+c_{0}
$$

for $t \in I$ a.e.

In our paper we prove the analogue of the above theorem for functions of two variables (the generalization of theorem 1.3).

TheOREM 3.2. If $v \in L^{2}\left(P^{2}, \mathbb{R}^{n}\right)$ and $w \in L^{1}\left(P^{2}, \mathbb{R}^{n}\right)$ are such that

$$
\iint_{P^{2}} v(s, t) \frac{\partial^{2} h}{\partial x \partial y}(s, t) d s d t=\iint_{P^{2}} w(s, t) h(s, t) d s d t
$$

for any $h \in A C_{0}^{(1,1), 2}\left(P^{2}, \mathbb{R}^{n}\right)$, then there exist functions $b_{0}(\cdot), c_{0}(\cdot) \in L^{2}\left(I, \mathbb{R}^{n}\right)$ and a constant $a_{00} \in \mathbb{R}^{n}$, such that

$$
v(s, t)=\int_{0}^{s} \int_{0}^{t} w(x, y) d x d y+b_{0}(t)+c_{0}(s)+a_{00} \quad \text { for }(s, t) \in P^{2} \text { a.e. }
$$


Proof. Let us put

$$
W(s, t)=\int_{0}^{s} \int_{0}^{t} w(x, y) d y d x
$$

for $(s, t) \in P^{2}$. Integrating by parts and using the assumptions, we obtain

$$
\begin{aligned}
\iint_{P^{2}} W(s, t) & \frac{\partial^{2} h}{\partial x \partial y}(s, t) d s d t=\iint_{P^{2}}\left(\int_{0}^{s} \int_{0}^{t} w(x, y) d y d x\right) \frac{\partial}{\partial x}\left(\frac{\partial h}{\partial y}\right)(s, t) d s d t \\
& =\int_{0}^{1} \int_{0}^{1}\left(\int_{0}^{s}\left(\int_{0}^{t} w(x, y) d y\right) d x\right) \frac{\partial}{\partial x}\left(\frac{\partial h}{\partial y}\right)(s, t) d s d t \\
& =\int_{0}^{1}\left[\int_{0}^{1} \int_{0}^{t} w(x, y) d y d x \frac{\partial h}{\partial y}(1, t)-\int_{0}^{1}\left(\int_{0}^{t} w(s, y) d y\right) \frac{\partial h}{\partial y}(s, t) d s\right] d t \\
& =\int_{0}^{1}\left(-\int_{0}^{1}\left(\int_{0}^{t} w(s, y) d y\right) \frac{\partial h}{\partial y}(s, t) d s\right) d t \\
& =-\int_{0}^{1}\left(\int_{0}^{1}\left(\int_{0}^{t} w(s, y) d y\right) \frac{\partial h}{\partial y}(s, t) d t\right) d s \\
& =-\int_{0}^{1}\left(\left(\int_{0}^{1} w(s, y) d y\right) h(s, 1)-\int_{0}^{1} w(s, t) h(s, t) d t\right) d s \\
& =\int_{0}^{1} \int_{0}^{1} w(s, t) h(s, t) d t d s=\iint_{P^{2}} v(s, t) \frac{\partial^{2} h}{\partial x \partial y}(s, t) d s d t .
\end{aligned}
$$

So,

$$
\iint_{P^{2}}(v(s, t)-W(s, t)) \frac{\partial^{2} h}{\partial x \partial y}(s, t) d s d t=0
$$

for any $h \in A C_{0}^{(1,1), 2}\left(P^{2}, \mathbb{R}^{n}\right)$.

Consequently, Theorem 1.3 yields the existence of functions $b_{0}(\cdot), c_{0}(\cdot) \in L^{2}\left(I, \mathbb{R}^{n}\right)$ and a constant $a_{00} \in \mathbb{R}^{n}$, such that

$$
v(s, t)-W(s, t)=b_{0}(t)+c_{0}(s)+a_{00}
$$

for $(s, t) \in P^{2}$ a.e., that is,

$$
v(s, t)=\int_{0}^{s} \int_{0}^{t} w(x, y) d x d y+b_{0}(t)+c_{0}(s)+a_{00}
$$

for $(s, t) \in P^{2}$ a.e. The proof is completed.

From Theorem 1.2 we can easily obtain

TheOREM 3.3. If $v \in L^{2}\left(I, \mathbb{R}^{n}\right)$ and $w \in L^{1}\left(I, \mathbb{R}^{n}\right)$ are such that

$$
\int_{I} v(t) h^{k}(t) d t=(-1)^{k} \int_{I} w(t) h(t) d t
$$

for any $h \in A C_{0}^{k, 2}\left(I, \mathbb{R}^{n}\right)$, then there exist constants $c_{0}, \ldots, c_{k-1} \in \mathbb{R}^{n}$ such that

$$
v(t)=\int_{0}^{t} \int_{0}^{t_{1}} \ldots \int_{0}^{t_{k-1}} w(\tau) d \tau d t_{k-1} \ldots d t_{1}+c_{k-1} t^{k-1}+\cdots+c_{1} t+c_{0}
$$

for $t \in I$ a.e. 
Proof. Let us put

$$
W(t)=\int_{0}^{t} \int_{0}^{t_{1}} \cdots \int_{0}^{t_{k-1}} w(\tau) d \tau d t_{k-1} \ldots d t_{1}
$$

for $t \in I$. Integrating by parts ( $k$ times) and using the assumptions, we obtain

$$
\begin{aligned}
\int_{I} W(t) h^{k}(t) d t & =\int_{I} W(t)\left(h^{k-1}\right)^{\prime}(t) d t \\
& =\int_{I}\left(\int_{0}^{t} \int_{0}^{t_{1}} \ldots \int_{0}^{t_{k-1}} w(\tau) d \tau t_{k-1} \ldots d t_{2} d t_{1}\right)\left(h^{k-1}\right)^{\prime}(t) d t \\
& =-\int_{I}\left(\int_{0}^{t} \int_{0}^{t_{2}} \ldots \int_{0}^{t_{k-1}} w(\tau) d \tau d t_{k-1} \ldots d t_{2}\right) h^{k-1}(t) d t \\
& =\ldots=(-1)^{k} \int_{I} w(t) h(t) d t=\int_{I} v(t) h^{k}(t) d t .
\end{aligned}
$$

So,

for any $h \in A C_{0}^{k, 2}\left(I, \mathbb{R}^{n}\right)$.

$$
\int_{I}(v(t)-W(t)) h^{k}(t) d t=0
$$

Consequently, Theorem 1.2 yields the existence of constants $c_{0}, \ldots, c_{k-1} \in \mathbb{R}^{n}$ such that

$$
v(t)-W(t)=c_{k-1} t^{k-1}+\ldots+c_{1} t+c_{0}
$$

for $t \in I$ a.e., that is,

$$
v(t)=\int_{0}^{t} \int_{0}^{t_{1}} \ldots \int_{0}^{t_{k-1}} w(\tau) d \tau d t_{k-1} \ldots d t_{1}+c_{k-1} t^{k-1}+\ldots+c_{1} t+c_{0}
$$

for $t \in I$ a.e.

In an analogous way we can obtain the following generalization of Theorem 2.1:

THEOREM 3.4. If $v \in L^{2}\left(P^{2}, \mathbb{R}^{n}\right)$ and $w \in L^{1}\left(P^{2}, \mathbb{R}^{n}\right)$ are such that

$$
\iint_{P^{2}} v(s, t) \frac{\partial^{k+l} h}{\partial x^{k} \partial y^{l}}(s, t) d s d t=(-1)^{k+l} \iint_{P^{2}} w(s, t) h(s, t) d s d t
$$

for any $h \in A C_{0}^{(k, l), 2}\left(P^{2}, \mathbb{R}^{n}\right)$, then there exist functions $b_{i}(\cdot) \in L^{2}\left(I, \mathbb{R}^{n}\right), i=0, \ldots$, $k-1, c_{j}(\cdot) \in L^{2}\left(I, \mathbb{R}^{n}\right), j=0, \ldots, l-1$, and constants $a_{i j} \in \mathbb{R}^{n}, i=0, \ldots, k-1$, $j=0, \ldots, l-1$, such that

$$
\begin{array}{r}
v(s, t)=\int_{0}^{s} \int_{0}^{s_{1}} \ldots \int_{0}^{s_{k-1}} \int_{0}^{t} \int_{0}^{t_{1}} \ldots \int_{0}^{t_{l-1}} w(\sigma, \tau) d \tau d t_{l-1} \ldots d t_{1} d \sigma d s_{k-1} \ldots d s_{1} \\
+\sum_{i=0}^{k-1} \sum_{j=0}^{l-1} a_{i j} s^{i} t^{j}+\sum_{i=0}^{k-1} b_{i}(t) s^{i}+\sum_{j=0}^{l-1} c_{j}(s) t^{j}
\end{array}
$$

for $(s, t) \in P^{2}$ a.e.

Proof. Let us put

$$
W(s, t)=\int_{0}^{s} \int_{0}^{s_{1}} \ldots \int_{0}^{s_{k-1}} \int_{0}^{t} \int_{0}^{t_{1}} \ldots \int_{0}^{t_{l-1}} w(\sigma, \tau) d \sigma d s_{k-1} \ldots d s_{1} d \tau d t_{l-1} \ldots d t_{1}
$$


for $(s, t) \in P^{2}$. Integrating by parts ( $k+l$ times) and using the assumptions, we get

$$
\begin{aligned}
& \iint_{P^{2}} W(s, t) \frac{\partial^{k+l} h}{\partial x^{k} \partial y^{l}}(s, t) d s d t \\
= & \iint_{P^{2}}\left(\int_{0}^{s} \int_{0}^{s_{1}} \ldots \int_{0}^{s_{k-1}} \int_{0}^{t} \int_{0}^{t_{1}} \ldots \int_{0}^{t_{l-1}} w(\sigma, \tau) d \tau d t_{l-1} \ldots d t_{1} d \sigma d s_{k-1} \ldots d s_{1}\right) \\
& \cdot \frac{\partial}{\partial x}\left(\frac{\partial^{k-1+l} h}{\partial x^{k-1} \partial y^{l}}\right)(s, t) d s d t \\
= & -\iint_{P^{2}}\left(\int_{0}^{s_{1}} \int_{0}^{s_{2}} \ldots \int_{0}^{s_{k-1}} \int_{0}^{t} \int_{0}^{t_{1}} \ldots \int_{0}^{t_{l-1}} w(\sigma, \tau) d \tau d t_{l-1} \ldots d t_{1} d \sigma d s_{k-1} \ldots d s_{2}\right) \\
& \cdot \frac{\partial^{k-1+l} h}{\partial x^{k-1} \partial y^{l}}(s, t) d s d t \\
= & \ldots=(-1)^{k+l} \iint_{P^{2}} w(s, t) h(s, t) d s d t=\iint_{P^{2}} v(s, t) \frac{\partial^{k+l} h}{\partial x^{k} \partial y^{l}}(s, t) d s d t .
\end{aligned}
$$

So,

$$
\iint_{P^{2}}(v(s, t)-W(s, t)) \frac{\partial^{k+l} h}{\partial x^{k} \partial y^{l}}(s, t) d s d t=0
$$

for any $h \in A C_{0}^{(k, l), 2}\left(P^{2}, \mathbb{R}^{n}\right)$.

Consequently, Theorem 2.1 yields the existence of functions $b_{i}(\cdot) \in L^{2}\left(I, \mathbb{R}^{n}\right), i=$ $0, \ldots, k-1, c_{j}(\cdot) \in L^{2}\left(I, \mathbb{R}^{n}\right), j=0, \ldots, l-1$, and constants $a_{i j} \in \mathbb{R}^{n}, i=0, \ldots, k-1$, $j=0, \ldots, l-1$, such that

$$
\begin{array}{r}
v(s, t)=\int_{0}^{s} \int_{0}^{s_{1}} \ldots \int_{0}^{s_{k-1}} \int_{0}^{t} \int_{0}^{t_{1}} \ldots \int_{0}^{t_{l-1}} w(\sigma, \tau) d \tau d t_{l-1} \ldots d t_{1} d \sigma d s_{k-1} \ldots d s_{1} \\
+\sum_{i=0}^{k-1} \sum_{j=0}^{l-1} a_{i j} s^{i} t^{j}+\sum_{i=0}^{k-1} b_{i}(t) s^{i}+\sum_{j=0}^{l-1} c_{j}(s) t^{j}
\end{array}
$$

for $(s, t) \in P^{2}$ a.e.

4. Applications to the coercive Dirichlet problem. Let us define in the class

$$
\begin{aligned}
A C^{(k, l), 2}\left(P^{2}, \mathbb{R}^{n}\right) & =\left\{h: P^{2} \rightarrow \mathbb{R}^{n} \mid \text { there exists a function } l \in L^{2}\left(P^{2}, \mathbb{R}^{n}\right)\right. \text { such that } \\
h\left(x_{1}, y_{1}\right) & =\int_{0}^{x_{1}} \int_{0}^{x_{2}} \cdots \int_{0}^{x_{k}} \int_{0}^{y_{1}} \int_{0}^{y_{2}} \ldots \int_{0}^{y_{l}} l(s, t) d t d y_{l} \ldots d y_{2} d s d x_{k} \ldots d x_{2} \\
& \text { for } \left.\left(x_{1}, y_{1}\right) \in P^{2}\right\}
\end{aligned}
$$

the following mapping

$$
\|\cdot\|: A C^{(k, l), 2}\left(P^{2}, \mathbb{R}^{n}\right) \ni h \mapsto\left(\iint_{P^{2}}\left|\frac{\partial^{k+l} h}{\partial x^{k} \partial y^{l}}(s, t)\right|^{2} d s d t\right)^{1 / 2} \in \mathbb{R}_{0}^{+} .
$$


It is easily seen that, for any $i=0, \ldots, k-1, j=0, \ldots, l-1$ and $\left(x_{1}, y_{1}\right) \in P^{2}$,

$$
\begin{aligned}
\left|\frac{\partial^{i+j} h}{\partial x^{i} \partial y^{j}}\left(x_{1}, y_{1}\right)\right| & =\left|\int_{0}^{x_{1}} \cdots \int_{0}^{x_{k-i}} \int_{0}^{y_{1}} \cdots \int_{0}^{y_{l-j}} \frac{\partial^{k+l} h}{\partial x^{k} \partial y^{l}}\right| \\
& \leq \int_{0}^{x_{1}} \cdots \int_{0}^{x_{k-i}} \int_{0}^{y_{1}} \cdots \int_{0}^{y_{l-j}}\left|\frac{\partial^{k+l} h}{\partial x^{k} \partial y^{l}}\right| \leq \iint_{P^{2}}\left|\frac{\partial^{k+l} h}{\partial x^{k} \partial y^{l}}\right| \\
& \leq\left(\iint_{P^{2}}\left|\frac{\partial^{k+l} h}{\partial x^{k} \partial y^{l}}(s, t)\right|^{2} d s d t\right)^{\frac{1}{2}}=\|h\| .
\end{aligned}
$$

From the above it follows that the mapping $\|\cdot\|$ restricted to the space $A C_{0}^{(k, l), 2}\left(P^{2}, \mathbb{R}^{n}\right)$ is the norm in this space.

Furthermore, $A C_{0}^{(k, l), 2}\left(P^{2}, \mathbb{R}^{n}\right)$ with this norm is a Banach space.

Indeed, let $\left(h_{n}\right)_{n \in \mathbb{N}}$ be a Cauchy sequence in $A C_{0}^{(k, l), 2}\left(P^{2}, \mathbb{R}^{n}\right)$. Then, of course, the sequence $\left(\frac{\partial^{k+l} h_{n}}{\partial x^{k} \partial y^{l}}\right)_{n \in \mathbb{N}}$ is a Cauchy sequence in $L^{2}\left(P^{2}, \mathbb{R}^{n}\right)$. So, there exists a function $l_{0} \in L^{2}\left(P^{2}, \mathbb{R}^{n}\right)$ that is the limit of the sequence $\left(\frac{\partial^{k+l} h_{n}}{\partial x^{k} \partial y^{l}}\right)_{n \in \mathbb{N}}$ in $L^{2}\left(P^{2}, \mathbb{R}^{n}\right)$. Now, if we put

$$
h_{0}\left(x_{1}, y_{1}\right)=\int_{0}^{x_{1}} \int_{0}^{x_{2}} \cdots \int^{x_{k}} \int_{0}^{y_{1}} \int_{0}^{y_{2}} \cdots \int_{0}^{y_{l}} l_{0}(s, t)
$$

for all $\left(x_{1}, y_{1}\right) \in P^{2}$, then we easily assert that $\left\|h_{n}-h_{0}\right\| \underset{n \rightarrow \infty}{\longrightarrow} 0$ and $h_{0} \in A C_{0}^{(k, l), 2}\left(P^{2}, \mathbb{R}^{n}\right)$ because

$$
\begin{aligned}
\left|\frac{\partial^{i+j} h_{0}}{\partial x^{i} \partial y^{j}}\left(1, y_{1}\right)\right| & \leq\left|\frac{\partial^{i+j} h_{0}}{\partial x^{i} \partial y^{j}}\left(1, y_{1}\right)-\frac{\partial^{i+j} h_{n}}{\partial x^{i} \partial y^{j}}\left(1, y_{1}\right)\right|+\left|\frac{\partial^{i+j} h_{n}}{\partial x^{i} \partial y^{j}}\left(1, y_{1}\right)\right| \\
& =\left|\frac{\partial^{i+j} h_{0}}{\partial x^{i} \partial y^{j}}\left(1, y_{1}\right)-\frac{\partial^{i+j} h_{n}}{\partial x^{i} \partial y^{j}}\left(1, y_{1}\right)\right|=\left|\frac{\partial^{i+j}\left(h_{0}-h_{n}\right)}{\partial x^{i} \partial y^{j}}\left(1, y_{1}\right)\right| \\
& \leq\left\|h_{0}-h_{n}\right\| \rightarrow 0
\end{aligned}
$$

and, analogously,

$$
\left|\frac{\partial^{i+j} h_{0}}{\partial x^{i} \partial y^{j}}\left(x_{1}, 1\right)\right| \leq\left\|h_{0}-h_{n}\right\| \rightarrow 0 .
$$

Let us observe that in the space $A C_{0}^{(k, l), 2}\left(P^{2}, \mathbb{R}^{n}\right)$ one can define the scalar product

$$
(g \mid h)=\iint_{P^{2}} \frac{\partial^{k+l} g}{\partial x^{k} \partial y^{l}}(s, t) \frac{\partial^{k+l} h}{\partial x^{k} \partial y^{l}}(s, t) d s d t .
$$

Of course, $\|\cdot\|$ is the norm determined by the above scalar product. So, $A C_{0}^{(k, l), 2}\left(P^{2}, \mathbb{R}^{n}\right)$ is a Hilbert space and, consequently, it is reflexive.

Now, we shall prove the analogue of [3, I.3. Lemma 2].

Lemma 4.1. If $h_{0} \in A C_{0}^{(k, l), 2}\left(P^{2}, \mathbb{R}^{n}\right)$ and $\left(h_{n}\right)_{n \in \mathbb{N}}$ is a sequence in $A C_{0}^{(k, l), 2}\left(P^{2}, \mathbb{R}^{n}\right)$ such that $h_{n} \underset{n \rightarrow \infty}{\rightarrow} h_{0}$ weakly in $A C_{0}^{(k, l), 2}\left(P^{2}, \mathbb{R}^{n}\right)$, then $h_{n} \underset{n \rightarrow \infty}{\rightrightarrows} h_{0}$ uniformly on $P^{2}$.

Pr o of. Since the sequence $\left(\left\|h_{n}\right\|\right)_{n \in \mathbb{N}}$ is bounded (cf. [2, III.24. Theorem 4]), therefore, in view of inequality (14), it is equibounded. 
Furthermore,

$$
\begin{aligned}
\mid F_{h_{n}}(P & =[\bar{x}, \overline{\bar{x}}] \times[\bar{y}, \overline{\bar{y}}])|=| h_{n}(\overline{\bar{x}}, \overline{\bar{y}})-h_{n}(\bar{x}, \overline{\bar{y}})-h_{n}(\overline{\bar{x}}, \bar{y})+h_{n}(\bar{x}, \bar{y}) \mid \\
& =\left|\int_{\bar{x}}^{\overline{\bar{x}}} \int_{\bar{y}}^{\overline{\bar{y}}} \frac{\partial^{2} h_{n}}{\partial x \partial y}\left(x_{1}, y_{1}\right) d x_{1} d y_{1}\right| \leq \int_{\bar{x}}^{\overline{\bar{x}}} \int_{\bar{y}}^{\overline{\bar{y}}}\left|\frac{\partial^{2} h_{n}}{\partial x \partial y}\left(x_{1}, y_{1}\right)\right| d x_{1} d y_{1} \\
& \leq \int_{\bar{x}}^{\overline{\bar{x}}} \int_{\bar{y}}^{\overline{\bar{y}}}\left(\iint_{P^{2}}\left|\frac{\partial^{k+l} h_{n}}{\partial x^{k} \partial y^{l}}\right|^{2}\right)^{\frac{1}{2}} d x_{1} d y_{1} \\
& =\left(\iint_{P^{2}}\left|\frac{\partial^{k+l} h_{n}}{\partial x^{k} \partial y^{l}}\right|^{2}\right)^{\frac{1}{2}} \int_{\bar{x}}^{\bar{x}} \int_{\bar{y}}^{\overline{\bar{y}}} 1 d x_{1} d y_{1} \\
& =\left\|h_{n}\right\| \mu_{2}(P) \leq c \mu_{2}(P) .
\end{aligned}
$$

for any $n \in \mathbb{N}$, where $c>0$ is a constant that bounds the sequence $\left(\left\|h_{n}\right\|\right)_{n \in \mathbb{N}}$.

So, the sequence $\left(h_{n}\right)_{n \in \mathbb{N}}$ is equiabsolutely continuous on $P^{2}$. Using the Ascoli-Arzela theorem for absolutely continuous functions of two variables (cf. [1]), we assert that the sequence $\left(h_{n}\right)_{n \in \mathbb{N}}$ possesses a subsequence $\left(h_{n_{k}}\right)_{k \in \mathbb{N}}$ uniformly convergent to some function $\bar{h}_{0}$ absolutely continuous on $P^{2}$. From this it follows that $h_{n_{k}} \underset{k \rightarrow \infty}{\rightarrow} \bar{h}_{0}$ in $C\left(P^{2}, \mathbb{R}^{n}\right)$. On the other side (because $A C_{0}^{(k, l), 2}\left(P^{2}, \mathbb{R}^{n}\right) \subset C\left(P^{2}, \mathbb{R}^{n}\right)$ and (14) holds), $h_{n_{k}} \underset{k \rightarrow \infty}{\rightarrow} h_{0}$ in $C\left(P^{2}, \mathbb{R}^{n}\right)$. So, $\bar{h}_{0}=h_{0}$, i.e. the subsequence $\left(h_{n_{k}}\right)_{k \in \mathbb{N}}$ converges uniformly to $h_{0}$ on $P^{2}$.

Let us observe that the sequence $\left(h_{n}\right)_{n \in \mathbb{N}}$ also converges uniformly to $h_{0}$ on $P^{2}$.

Indeed, let us assume that this is not true. One can choose some subsequence $\left(h_{n_{l}}\right)_{l \in \mathbb{N}}$ such that

$$
\max \left\{\left|h_{n_{l}}(x, y)-h_{0}(x, y)\right|,(x, y) \in P^{2}\right\}>\varepsilon
$$

for any $l \in \mathbb{N}$, where $\varepsilon>0$ is some fixed constant. Since $h_{n_{l}} \underset{l \rightarrow \infty}{\rightarrow} h_{0}$ in $A C_{0}^{(k, l), 2}\left(P^{2}, \mathbb{R}^{n}\right)$, therefore, repeating the reasoning from the first part of this proof, we assert that the subsequence $\left(h_{n_{l}}\right)_{l \in \mathbb{N}}$ possesses a subsequence uniformly convergent to $h_{0}$ on $P^{2}$. The contradiction obtained completes the proof.

Now, let us consider in the space $A C_{0}^{(k, l), 2}\left(P^{2}, \mathbb{R}^{n}\right)$ the following functional:

$$
f(z)=\iint_{P^{2}}\left(\frac{1}{2}\left|\frac{\partial^{k+l} z}{\partial x^{k} \partial y^{l}}(x, y)\right|^{2}+F(x, y, z(x, y))\right) d x d y
$$

where $F: P^{2} \times \mathbb{R}^{n} \rightarrow \mathbb{R}$ is continuous and such that the partial derivatives $\frac{\partial F}{\partial z_{i}}, i=$ $1, \ldots, n$, exist and are continuous on $P^{2} \times \mathbb{R}^{n}$.

It is easy to see that $f$ is Gâteaux-differentiable at each point $z \in A C_{0}^{(k, l), 2}\left(P^{2}, \mathbb{R}^{n}\right)$, and

$$
\partial f(z)(h)=\iint_{P^{2}}\left(\frac{\partial^{k+l} z(x, y)}{\partial x^{k} \partial y^{l}} \frac{\partial^{k+l} h(x, y)}{\partial x^{k} \partial y^{l}}+\nabla F(x, y, z(x, y)) h(x, y)\right) d x d y
$$

for any $h \in A C_{0}^{(k, l), 2}\left(P^{2}, \mathbb{R}^{n}\right)$, where $\nabla F=\left(\frac{\partial F}{\partial z_{1}}, \ldots, \frac{\partial F}{\partial z_{n}}\right)$.

Furthermore, we have 
LEMMA 4.2. If there exist constants $a<1, b \geq 0, c \geq 0$ such that

$$
F(x, y, z) \geq-a \frac{|z|^{2}}{2}-b|z|-c \quad \text { for }(x, y, z) \in P^{2} \times \mathbb{R}^{n},
$$

then the functional $f$ is coercive and weakly l.s.c. on $A C_{0}^{(k, l), 2}\left(P^{2}, \mathbb{R}^{n}\right)$.

Proof. For any $z \in A C_{0}^{(k, l), 2}\left(P^{2}, \mathbb{R}^{n}\right)$, we have

$$
\begin{aligned}
f(z) & =\iint_{P^{2}}\left(\frac{1}{2}\left|\frac{\partial^{k+l} z}{\partial x^{k} \partial y^{l}}(x, y)\right|^{2}+F(x, y, z(x, y))\right) d x d y \\
& \geq \iint_{P^{2}}\left(\frac{1}{2}\left|\frac{\partial^{k+l} z}{\partial x^{k} \partial y^{l}}(x, y)\right|^{2}-\frac{a}{2}|z(x, y)|^{2}-b|z(x, y)|-c\right) d x d y \\
& =\frac{1}{2}\|z\|^{2}-\frac{a}{2} \iint_{P^{2}}|z(x, y)|^{2}-b \iint_{P^{2}}|z(x, y)|-c \\
& \geq \frac{1}{2}\|z\|^{2}-\frac{a}{2} \iint_{P^{2}}\|z\|^{2}-b \iint_{P^{2}}\|z\|-c \\
& =\frac{1}{2}\|z\|^{2}-\frac{a}{2}\|z\|^{2}-b\|z\|-c \\
& =\frac{1-a}{2}\|z\|^{2}-b\|z\|-c .
\end{aligned}
$$

This means that $f(z) \rightarrow+\infty$ when $\|z\| \rightarrow+\infty$, i.e. $f$ is coercive.

To prove that $f$ is weakly l.s.c., assume that the sequence $\left(z_{m}\right)_{m \in \mathbb{N}}$ converges weakly to $z_{0}$ in $A C_{0}^{(k, l), 2}\left(P^{2}, \mathbb{R}^{n}\right)$. From this and from the inequality

it follows that

$$
\begin{aligned}
0 \leq & \iint_{P^{2}}\left|\frac{\partial^{k+l} z_{m}}{\partial x^{k} \partial y^{l}}(x, y)-\frac{\partial^{k+l} z_{0}}{\partial x^{k} \partial y^{l}}(x, y)\right|^{2} d x d y \\
= & \iint_{P^{2}}\left|\frac{\partial^{k+l} z_{m}}{\partial x^{k} \partial y^{l}}\right|^{2}-2 \iint_{P^{2}} \frac{\partial^{k+l} z_{m}}{\partial x^{k} \partial y^{l}}(x, y) \frac{\partial^{k+l} z_{0}}{\partial x^{k} \partial y^{l}}(x, y) \\
& +\iint_{P^{2}}\left|\frac{\partial^{k+l} z_{0}}{\partial x^{k} \partial y^{l}}(x, y)\right|^{2}
\end{aligned}
$$

$$
\liminf _{m \rightarrow \infty} \iint_{P^{2}}\left|\frac{\partial^{k+l} z_{m}}{\partial x^{k} \partial y^{l}}(x, y)\right|^{2} \geq \iint_{P^{2}}\left|\frac{\partial^{k+l} z_{0}}{\partial x^{k} \partial y^{l}}(x, y)\right|^{2}
$$

Lemma 4.1 implies the equality

$$
\lim _{m \rightarrow \infty} \iint_{P^{2}} F\left(x, y, z_{m}(x, y)\right) d x d y=\iint_{P^{2}} F\left(x, y, z_{0}(x, y)\right) d x d y .
$$

Consequently,

$$
\liminf _{m \rightarrow \infty} f\left(z_{m}\right) \geq f\left(z_{0}\right)
$$

The proof is completed.

From the above lemma and [3, I.2. Proposition 2] it follows that there exists a point $z_{0} \in A C_{0}^{(k, l), 2}\left(P^{2}, \mathbb{R}^{n}\right)$ such that

$$
f\left(z_{0}\right)=\min \left\{f(z) \mid z \in A C_{0}^{(k, l), 2}\left(P^{2}, \mathbb{R}^{n}\right)\right\} .
$$


In view of the Gâteaux-differentiability of $f$, this means that

$$
\partial f\left(z_{0}\right)=0
$$

i.e.

$$
\iint_{P^{2}} \frac{\partial^{k+l} z_{0}}{\partial x^{k} \partial y^{l}}(x, y) \frac{\partial^{k+l} h}{\partial x^{k} \partial y^{l}}(x, y)=-\iint_{P^{2}} \nabla F\left(x, y, z_{0}(x, y)\right) h(x, y)
$$

for any $h \in A C_{0}^{(k, l), 2}\left(P^{2}, \mathbb{R}^{n}\right)$. From theorem 3.4 it follows that there exist functions $b_{i}(\cdot) \in L^{2}\left(I, \mathbb{R}^{n}\right), i=0, \ldots, k-1, c_{j}(\cdot) \in L^{2}\left(I, \mathbb{R}^{n}\right), j=0, \ldots, l-1$, and constants $a_{i j} \in \mathbb{R}^{n}, i=0, \ldots, k-1, j=0, \ldots, l-1$, such that

$$
\text { (16) } \begin{aligned}
\frac{\partial^{k+l} z_{0}}{\partial x^{k} \partial y^{l}}(x, y)= & \int_{0}^{x} \int_{0}^{x_{2}} \cdots \int_{0}^{x_{k}} \int_{0}^{y} \int_{0}^{y_{2}} \cdots \int_{0}^{y_{l}}(-1)^{k+l-1} \nabla F\left(x, y, z_{0}(x, y)\right) \\
& +\sum_{i=0}^{k-1} \sum_{j=0}^{l-1} a_{i j} x^{i} y^{j}+\sum_{i=0}^{k-1} b_{i}(y) x^{i}+\sum_{j=0}^{l-1} c_{j}(x) y^{j}
\end{aligned}
$$

for $(x, y) \in P^{2}$ a.e., thus for $(x, y) \in \operatorname{Int} P^{2}$ a.e.

From (15) it follows that $\frac{\partial^{k+l} z_{0}}{\partial x^{k} \partial y^{l}}$ has a weak derivative of order $(k, l)$ equal to $(-1)^{k+l-1} \nabla F\left(x, y, z_{0}(x, y)\right)$. So, from the fact that the weak derivative of order $(k, l)$ of the function $z_{0}$ is equal to $\frac{\partial^{k+l} z_{0}}{\partial x^{k} \partial y^{l}}$ (the classical derivative), i.e.

$$
D^{(k, l)} z_{0}(x, y)=\frac{\partial^{k+l} z_{0}}{\partial x^{k} \partial y^{l}}(x, y)
$$

for $(x, y) \in \operatorname{Int} P^{2}$ a.e., we have

$$
D^{(2 k, 2 l)} z_{0}(x, y)+(-1)^{k+l} \nabla F\left(x, y, z_{0}(x, y)\right)=0
$$

for $(x, y) \in \operatorname{Int} P^{2}$ a.e. Denoting $D^{(2 k, 2 l)} z_{0}$ by $\frac{\partial^{2 k+2 l} z_{0}}{\partial x^{2 k} \partial y^{2 l}}$, we can write

$$
\frac{\partial^{2 k+2 l} z_{0}}{\partial x^{2 k} \partial y^{2 l}}(x, y)+(-1)^{k+l} \nabla F\left(x, y, z_{0}(x, y)\right)=0
$$

for $(x, y) \in \operatorname{Int} P^{2}$ a.e.

We have thus proved

TheOREM 4.3. If a function $F: P^{2} \times \mathbb{R}^{n} \rightarrow \mathbb{R}$ is continuous, possesses continuous partial derivatives $\frac{\partial F}{\partial z_{i}}, i=1, \ldots, n$, and there exist constants $a<1, b \geq 0, c \geq 0$ such that

$$
F(x, y, z) \geq-a \frac{|z|^{2}}{2}-b|z|-c
$$

for $(x, y, z) \in P^{2} \times \mathbb{R}^{n}$, then the system

$$
\frac{\partial^{2 k+2 l} z}{\partial x^{2 k} \partial y^{2 l}}+(-1)^{k+l} \nabla F(x, y, z)=0
$$

a.e. in Int $P^{2}$ possesses a solution $z$ (in a weak sense) in the space $A C_{0}^{(k, l), 2}\left(P^{2}, \mathbb{R}^{n}\right)$ such that $\frac{\partial^{k+l} z_{0}}{\partial x^{k} \partial y^{l}}$ has the form (16). 
Rem ark. From the definition of the space $A C_{0}^{(k, l), 2}\left(P^{2}, \mathbb{R}^{n}\right)$ it follows that the solution $z$ of system (17) satisfies the boundary conditions

$$
\left.\frac{\partial^{i+j} z}{\partial x^{i} \partial y^{j}}\right|_{\partial P^{2}} \equiv 0
$$

for $i=0, \ldots, k-1, j=0, \ldots, l-1$.

\section{References}

[1] D. Idczak and S. Walczak, On the existence of a solution for some distributed optimal control hyperbolic system, to appear in International Journal of Mathematics and Mathematical Sciences, University of Central Florida.

[2] L. A. Lusternik and W. I. Sobolew, Elements of Functional Analysis, Warsaw 1959, (Polish).

[3] J. Mawhin, Problèmes de Dirichlet Variationnels Non-Linéaires, L'Université de Montréal, 1987.

[4] S. Walczak, On some generalization of the fundamental lemma and its application to differential equations, Bull. Soc. Math. Belg. 45(3) ser. B (1993).

[5] S. Walczak, On the Du Bois-Reymond lemma for functions of several variables, ibid. 